\title{
Chronic loneliness: neurocognitive mechanisms and interventions
}

\section{Authors:}

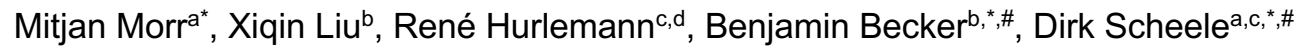

\begin{abstract}
Affiliations:
a Division of Medical Psychology, Department of Psychiatry and Psychotherapy, University Hospital Bonn, 53127 Bonn, Germany

${ }^{b}$ Clinical Hospital of Chengdu Brain Science Institute, School of Life Science and Technology,

University of Electronic Science and Technology of China, 611731, Chengdu, China

${ }^{\mathrm{c}}$ Department of Psychiatry, School of Medicine \& Health Sciences, University of Oldenburg, 26129

Oldenburg, Germany

${ }^{\mathrm{d}}$ Research Center Neurosensory Science, University of Oldenburg, 26129 Oldenburg, Germany

\# These authors contributed equally
\end{abstract}

${ }^{*}$ Corresponding authors:

Mitjan Morr, M.Sc.

Division of Medical Psychology

University Hospital Bonn

Venusberg-Campus 1

53127 Bonn, Germany

Phone: +49 (0)228-287 19578

E-mail: Mitjan.Morr@ukbonn.de

Benjamin Becker, Ph.D.

Clinical Hospital of Chengdu Brain Science Institute

School of Life Science and Technology

University of Electronic Science and Technology

Xiyuan Ave 2006

611731 Chengdu, China

Phone: +86 (0) 185-8407 0309

E-mail: ben_becker@gmx.de

Dirk Scheele, Ph.D.

Department of Psychiatry

University of Oldenburg

Hermann-Ehlers-Str. 7

26160 Bad Zwischenahn, Germany

Phone: +49-(0)441-9615-1508

E-mail: Dirk-Scheele@gmx.de

Article information:

Type of submission: Editorial Letter

Number of tables: 0

Number of figures: 2 (separate files)

Number of words: 2723

Keywords: Cognitive biases, chronic loneliness, fMRI, loneliness intervention, neural mechanisms 


\section{Main text}

2 Loneliness has been associated with detrimental effects on mental and physical health and is

3 increasingly recognized as a critical public health issue which may be further exacerbated by societal challenges such as increasing urbanization, an aging society as well as the COVID19 pandemic. A recent clinical study published in Psychotherapy and Psychosomatics has demonstrated that an internet-based cognitive behavioral therapy can significantly reduce loneliness and such a preventive intervention may be co-opted to target suicidality in the elderly $[1,2]$. As such, it is now an opportune time to review current conceptualization of chronic loneliness, its detrimental consequences and potential neurocognitive mechanisms as well as initial treatment strategies.

Loneliness is not a clinical diagnosis, but a psychological state with detrimental effects on physiological and mental health if it is experienced chronically. Prevalence estimates vary depending on the assessment criteria, but representative samples surveyed before the onset of the COVID-19 pandemic showed that $22 \%$ of inhabitants in the United States and $23 \%$ in Britain always or often feel lonely [3]. Loneliness can occur at any life stage, but elevated levels have been observed during late adolescence and in elderly people [4]. Various lines of research also indicate that the extended lockdowns and necessary social isolation during the COVID-19 pandemic have increased not only feelings of loneliness but also depression and suicidal ideation [5-7]. However, of note, loneliness is a subjective feeling which is distinct from objective social isolation $[8,9]$. It is possible to have a large and diverse social network and feel lonely, and vice versa, to live a life with only a few meaningful social connections and experience no loneliness at all. Therefore, loneliness can be best described as a discrepancy between desired and actual social connectedness [10]. In humans as a social species loneliness may have evolved as an adaptive function and evolutionary coping strategy to promote behavioral changes, which increase the chance of survival [11]. Loneliness can be seen as a social equivalent to hunger, such that the feeling of loneliness triggers the need to form new social relationships, in the same way as hunger triggers the need to eat [12-14]. If 
loneliness is an evolutionary signal to form social bonds, the question of why some people stay lonely over extended periods of time arises. Current models of loneliness postulate that lonely individuals exhibit negative social biases which paradoxically lead to even more withdrawal from social connections [15]. Clearly, the effects of acute loneliness are distinct from the impact of chronic loneliness $[16,17]$. For instance, a recent study found that chronic loneliness was associated with a greater preferred interpersonal distance, whereas acute loneliness was related to smaller preferred distances [18], possibly reflecting the evolutionary desire to form social bonds. Although acute social exclusion elicits activations in neural pathways overlapping with those mediating physical pain and may lead to severe distress [19,20], chronic loneliness exerts more harmful effects, such as strongly increased mortality in comparison to acute social isolation [21]. Given that the COVID-19 pandemic and the necessary measures of social distancing may facilitate the transition from acute to chronic loneliness [22], interventions in vulnerable populations may help prevent detrimental health consequences of loneliness.

\section{Detrimental Health Consequences of Loneliness}

Accumulating evidence from different lines of research convergently indicates the detrimental impact of chronic loneliness and perceived social isolation on both, somatic and mental health. A number of studies have established associations between chronic loneliness and increased morbidity and mortality mirroring the negative impact of well-established risk factors such as obesity or smoking. Thus, loneliness and social disconnection are increasingly recognized as a major public health concern [23-26]. Increasing evidence suggests associations between chronic loneliness and an impaired integrity of the immune system, including reduced numbers of natural killer cells [27, 28] and diminished immune responses to acute stressors [29] in lonely individuals. Chronic loneliness has also been linked to heightened blood pressure [30,31] and an increased risk for coronary heart diseases and stroke [32]. In addition, feelings of social isolation are a risk factor for obesity [33-35] and impaired sleep quality [36, 37]. Sleep deprivation in turn can trigger feelings of loneliness, starting a self-reinforcing cycle of social 
withdrawal [38]. Importantly, the detrimental effects of loneliness are not restricted to somatic disorders, but extend to mental health. Perceived social isolation has been identified as a significant predictor for cognitive decline in dementia and Alzheimer disease $[39,40]$ and is associated with higher levels of depressive symptoms [41, 42], anxiety [43, 44], and psychosocial stress [45]. Furthermore, patients with substance abuse [46-48], borderline personality disorder [49, 50], and schizoid personality disorder [51] report more loneliness and social disconnection than healthy controls. In addition, loneliness is a potential risk factor for post-traumatic stress disorder $[52,53]$ and enhances intrusive thoughts after trauma exposure $[54,55]$. Overall, loneliness and social isolation are critical risk factors for several somatic and mental disorders and thus should be considered in therapeutic protocols. The development of neurobiologically informed interventions for loneliness critically requires a better understanding of the brain structural and functional neural changes related to chronic feeling of social isolation.

\section{Brain Structural Adaptations Associated with Loneliness}

Prolonged periods of social isolation have been linked to broad changes in brain morphology. For instance, participants of a 14-months long Antarctica expedition exhibited significant reductions in brain-derived neurotrophic factor concentrations and gray matter volume in the dorsolateral and orbitofrontal cortex and hippocampus compared to controls [56]. While these findings are consistent with animal studies showing an association between social isolation and hippocampal neurogenesis [57], it is also conceivable that the expedition-related changes are a byproduct of sensory deprivation. Previous studies also observed that larger and more diverse social networks positively correlate with amygdala volume [58], but a recent study failed to replicate this association [59]. Along these lines, a rare patient with bilateral amygdala damage showed a normal size and complexity of her social network [60], indicating that an intact amygdala is not necessary to maintain social relationships or at least can be compensated for [61]. Several years after the first assessment of the social network, the 
woman with amygdala lesion developed severe treatment-resistant depression along with a reduction in the size of her social network, and she reported strong feelings of loneliness [62], demonstrating that the experience of loneliness may not require an intact amygdala either. However, recent large scale brain morphology studies suggest that there are sex-dependent brain volume effects of loneliness, especially in the amygdala and the ventromedial prefrontal cortex (vmPFC) [63]. Smaller amygdala volumes were detected for lonely men, but not lonely women, and this pattern was reversed for the vmPFC volume. Thus, prospective longitudinal studies are required to monitor sex-specific morphological changes that accompany chronic loneliness. Sex and loneliness interactions are not restricted to brain structural effects. It has been found that lonely individuals display stronger functional communication in the default mode network (DMN) and this loneliness-related effect was more prominent in men than women [64].

Furthermore, individual differences in loneliness correlated with gray matter density in the left posterior superior temporal sulcus and this association was mediated by social perception skills [65]. Interestingly, the correlation remained significant after controlling for trait anxiety and social network size, thus providing further support for the notion that loneliness and social anxiety are characterized by distinct neural phenotypes [66] and for the dissociation of loneliness and social isolation. Importantly, loneliness has also been linked to altered neural processing in various neurocognitive domains (cf. Figure 1), including negative cognitive biases, sensory processing, executive functioning, reward-related processes, and memory.

\section{Negative Cognitive Biases}

It has been hypothesized that the maintenance of loneliness is fueled by negative cognitive biases which make positive social interactions less rewarding and may foster even more social withdrawal $[13,67]$. Mechanistically, lonely individuals may be more likely to perceive social stimuli as threatening and to evaluate themselves and others more negatively [15]. Feelings of alienation may result from larger self-other dissimilarity of activation patterns in the medial 
prefrontal cortex [68]. Furthermore, loneliness is associated with reduced interpersonal trust and a preference for larger social distances from unfamiliar others and this behavioral phenotype is paralleled by reduced trust-associated activity in the anterior insula. Importantly, blunted functional connectivity between the anterior insula and occipito-parietal regions predicts diminished affective and oxytocinergic responses to positive social interaction [69]. Given that the anterior insula plays a key role in self-awareness and interoceptive processing [70], we hypothesize that the negative cognitive biases in loneliness are mediated by an external attention focus due to reduced generation of or sensitivity to internal physiological signals in social situations [71]. Further supporting evidence for this notion comes from a study showing that insula responses to emotional faces mediate the association between alexithymia and subjective isolation stress [45]. Increased functional connectivity of the DMN [64] and overall increased network integration between the DMN and the attentional and visual networks in lonely subjects [72] may reflect exaggerated rumination during rest [73]. In addition, loneliness may affect synchronization during social interactions, such that lonely people may require stronger activation of their observation execution system including the inferior frontal gyrus (IFG) and the inferior parietal lobule for alignment to compensate for some deficiency in their synchronization ability [74]. Further studies are warranted to probe possible causal pathways of how disrupted interoceptive processes and impaired synchronization may lead to social withdrawal and the chronicity of loneliness.

\section{Sensory Processing}

Loneliness-induced hypervigilance can be observed in a shift of visual and auditory attention to negative or threating stimuli. These changes in sensory processing could be induced by alterations in the dorsal and ventral attention networks $[72,75]$. Furthermore, there appears to be a bidirectional relationship between tactile processing and loneliness. Touch deprivation during COVID-19-related restrictions has been linked to higher anxiety and greater loneliness [76], but loneliness also positively correlated with touch avoidance [77]. The excitatory 
transcranial direct current stimulation to the right IFG slowed responses to observed touch in lonely individuals [77], indicating that the IFG may contribute to the perpetuation of loneliness by enhancing the avoidance of positive social cues. Likewise, olfactory impairment can severely disrupt close relationships [78]. Loneliness is higher in participants who experienced childhood maltreatment, which correlates with amygdala hyperreactivity and hippocampal deactivation in response to social stress odors [79]. Whether and how loneliness may affect the sensory integration of multiple modalities is still elusive.

\section{Executive Functioning and Cognitive Control}

Loneliness is a risk factor for cognitive decline and dementia in older individuals, but evidence for an association between loneliness and impaired executive functioning across the life span is scarce. Attentional regulation was reduced in lonely individuals in a dichotic listening task [80] and loneliness was associated with significantly reduced odds of physical activity [33], which may reflect decreased cognitive control. Acute social exclusion has been found to impair self-regulation, possibly because it makes excluded people less inclined to make the effort to control impulses $[81,82]$. It has been hypothesized that reduced functional connectivity of the right middle/superior frontal gyrus to the cingulo-opercular network during rest may reflect diminished executive functioning in loneliness [83], but future studies are needed to test a causal relationship between loneliness and executive functioning.

\section{Reward-Related Processes}

The activation patterns evoked by acute social isolation in the ventral tegmental area is similar to the craving-related activation pattern observed after fasting [14]. By contrast, dissociable responses were evident in the striatum, with fasting enhancing responses to food cues in the nucleus accumbens and social isolation increasing responses to social cues in the caudate nucleus. Cacioppo et al. showed reduced ventral striatum (VS) activity in lonely individuals 
while viewing pleasant pictures with social connotation [84], but other studies found no significant correlation between loneliness and VS responses to pleasant social stimuli [85], nor between loneliness and striatal dopamine synthesis capacity in healthy controls or patients with autism spectrum disorder [86]. These contradictory findings may be reconciled by taking the familiarity of the social context into account. For instance, another functional magnetic resonance imaging study reported selectively increased VS responses to images of close others compared to strangers in lonely individuals, possibly reflecting fear of alienation or rejection [12].

\section{Memory and Working Memory}

In line with the above-mentioned association between loneliness and cognitive decline, several studies have reported loneliness-related declines in episodic, semantic, and working memory in older adults [87-89]. In patients with major depressive disorder, loneliness had no significant effect on working memory performance, but it was linked to increased functional connectivity between the dorsolateral prefrontal cortex and inferior parietal cortex, indicating that loneliness may be associated with altered neural regulatory functioning in self-referential processing [90]. Of note, a recent study found that loneliness may influence trauma memory in a sex-dependent manner. Specifically, lonely men, but not lonely women, exhibited more intrusive thoughts after experimental trauma and this phenotype was related to amygdala hyperreactivity during both fear conditioning and habituation processes, suggesting that the limbic system is a potential target for interventions that increase social connectedness [55].

\section{Therapeutic Interventions for Loneliness and Integration with Neurocognitive}

\section{Mechanisms}

A recent meta-analysis showed that psychological interventions were more effective than measures to increase access to other people to improve the perceived quality of social 
connections [91]. For example, cognitive-behavioral therapies targeting maladaptive cognition can reduce loneliness levels and the elevated blood pressure associated with loneliness in older individuals [92, 93]. Furthermore, mindfulness training has been demonstrated to be effective in reducing loneliness and related pro-inflammatory gene expression [94-96]. Further studies have focused on designing and evaluating internet- or tele-delivered approaches that may facilitate more scalable and accessible interventions for chronic loneliness. A recent randomized controlled trial compared internet-based cognitive behavioral therapy (ICBT) and internet-based interpersonal psychotherapy (IIPT) and demonstrated a significantly greater efficacy of ICBT than IIPT in reducing loneliness [2]. Similarly, a short-term tele-delivered intervention that aimed at facilitating social connectedness showed promising results in older adults by reducing feelings of loneliness and depression [97]. Overall, there is growing evidence that behavioral and psychological interventions targeting loneliness are an effective way to increase the feeling of social connectedness and additionally reduce harmful health effects. Despite increasing evidence demonstrating the efficacy of behavioral interventions for loneliness, the brain-based mechanisms mediating interventional effects have not been examined. Future prospective studies are needed to differentiate predisposing brain alterations that render individuals vulnerable to chronic loneliness from alterations as a consequence of prolonged loneliness and those that normalize during the course of successful treatment. Moreover, a better understanding of the neurocognitive mechanisms mediating chronic loneliness opens up novel opportunities to enhance the efficacy of loneliness interventions by targeting the underlying brain circuits. Loneliness-related functional and structural brain changes are evident in various neural circuits of social and affective brain systems, including limbic regions such as the amygdala, hippocampus and the anterior insula, as well as striatal, prefrontal, and temporal regions (cf. Figure 2). Alterations in the underlying brain circuits have been associated with detrimental effects of loneliness in various functional domains, which appear to be distinct from the consequences of depression [98] and social anxiety [66]. Therapy outcomes may be improved when interventions focus on multiple functional domains and the related neural targets. For instance, accumulating evidence from basic research and 
216 proof-of-concept studies suggests that targeting hormonal systems such as the oxytocin or

217 vasopressin system may have the potential to facilitate social functioning in relevant domains

218 in both, healthy individuals as well as patients with mental disorders [99]. A single intranasal

219 dose of oxytocin reduced aversive anticipation in high anxious individuals [100] and prevented

220 sensitization towards angry faces [101] via reducing amygdala reactivity. Furthermore,

221 oxytocin was found to enhance approach behavior towards positive social stimuli by

222 modulating responsivity of the anterior insula [102, 103]. Both, single-dose administrations of

223 oxytocin and vasopressin may enhance the salience of social stimuli and decrease reactivity

224 towards negative social feedback [104, 105]. Although neuropeptide treatment effects in these

225 domains may vary as a function of dosage [106, 107], treatment expectation [108-110], and

226 sex [111-113], the adjunct administration in combination with behavioral interventions may

227 represent a promising venue to enhance the efficacy of loneliness interventions.

229 Conclusion

230 Taken together, loneliness is a crucial and modifiable risk factor for physical and mental health.

231 A better understanding of the neural underpinnings of social (dis)connectedness can help

232 boost the efficiency of loneliness interventions not only in healthy participants but also in 233 patients with mental disorders. 
236 The authors report no competing biomedical financial interests or personal affiliations in 237 connection with the content of this manuscript.

239 Funding Source

240 Dr. Scheele is supported by an Else-Kröner-Fresenius-Stiftung grant (2017_A35).

241

\section{Author Contributions}

243 M.M., X.L., R.H., B.B and D.S. all made substantial contributions to the conception of this 244 paper. M.M. and D.S. drafted this paper, and X.L. and B.B. contributed critical revisions for 245 intellectual content. All of the authors had final approval of all of the submitted versions, and 246 all are in agreement to be accountable for all aspects of this work. 


\section{References}

1 Siu HC, Lee S-H, Au JS, Lo AP-K, Huang C-M, Tsai Y-F, et al. Loneliness and Major Depressive Disorder in the Elderly with a History of Suicidal Ideation or Attempt: A Comment on "Therapist-Guided Internet-Based Treatments for Loneliness" by Käll et al. Psychother Psychosom. 2021:1-3.

2 Käll A, Bäck M, Welin C, Åman H, Bjerkander R, Wänman M, et al. Therapist-Guided Internet-Based Treatments for Loneliness: A Randomized Controlled Three-Arm Trial Comparing Cognitive Behavioral Therapy and Interpersonal Psychotherapy. Psychother Psychosom. 2021;90:351-358.

3 DiJulio B, Hamel L, Munana C, Brodie M. Loneliness and Social Isolation in the United States, the United Kingdom, and Japan: An International Survey: Kaiser Family Foundation. 2018.

$4 \quad$ Luhmann M, Hawkley LC. Age differences in loneliness from late adolescence to oldest old age. Developmental psychology 2016;52:943.

$5 \quad$ Killgore WDS, Cloonan SA, Taylor EC, Dailey NS. Loneliness: A signature mental health concern in the era of COVID-19. Psychiatry Res. 2020;290:113117.

$6 \quad$ Killgore WDS, Cloonan SA, Taylor EC, Lucas DA, Dailey NS. Loneliness during the first half-year of COVID-19 Lockdowns. Psychiatry Res. 2020;294:113551.

7 Killgore WDS, Cloonan SA, Taylor EC, Miller MA, Dailey NS. Three months of loneliness during the COVID-19 lockdown. Psychiatry Res. 2020;293:113392.

$8 \quad$ Cacioppo JT, Cacioppo S. The growing problem of loneliness. Lancet 2018;391:426.

9 Hawkley LC, Cacioppo JT. Loneliness Matters: A Theoretical and Empirical Review of Consequences and Mechanisms. Ann Behav Med. 2010;40:218-227.

10 Peplau LA, Perlman D. Loneliness: A sourcebook of current theory, research and therapy. John Wiley \& Sons Inc. 1982.

11 Cacioppo JT, Cacioppo S, Boomsma DI. Evolutionary mechanisms for loneliness. Cogn Emot. 2014;28:3-21.

12 Inagaki TK, Muscatell KA, Moieni M, Dutcher JM, Jevtic I, Irwin MR, et al. Yearning for connection? Loneliness is associated with increased ventral striatum activity to close others. Soc Cogn Affect Neurosci. 2016;11:1096-101.

13 Qualter P, Vanhalst J, Harris R, Van Roekel E, Lodder G, Bangee M, et al. Loneliness across the life span. Perspect Psychol Sci. 2015;10(2):250-64.

14 Tomova L, Wang KL, Thompson T, Matthews GA, Takahashi A, Tye KM, et al. Acute social isolation evokes midbrain craving responses similar to hunger. Nat Neurosci. 2020;23(12):1597-1605.

15 Spithoven AWM, Bijttebier P, Goossens L. It is all in their mind: A review on information processing bias in lonely individuals. Clin Psychol Rev. 2017;58:97-114.

16 Roddick CM, Chen FS. Effects of Chronic and State Loneliness on Heart Rate Variability in Women. Ann Behav Med. 2021;55:460-475.

17 Vanhalst J, Soenens B, Luyckx K, Van Petegem S, Weeks MS, Asher SR. Why do the lonely stay lonely? Chronically lonely adolescents' attributions and emotions in situations of social inclusion and exclusion. J Pers Soc Psychol. 2015;109:932.

18 Saporta N, Scheele D, Lieberz J, Stuhr-Wulff F, Hurlemann R, Shamay-Tsoory SG. Opposing Association of Situational and Chronic Loneliness with Interpersonal Distance. Brain Sci. 2021;11:1135.

19 Eisenberger NI, Lieberman MD, Williams KD. Does Rejection Hurt? An fMRI Study of Social Exclusion. Science. 2003;302:290-292.

20 MacDonald G, Leary MR. Why does social exclusion hurt? The relationship between social and physical pain. Psychol Bull. 2005;131:202.

21 Shiovitz-Ezra S, Ayalon L. Situational versus chronic loneliness as risk factors for allcause mortality. Int Psychogeriatr. 2010;22:455-462.

22 Luchetti M, Lee JH, Aschwanden D, Sesker A, Strickhouser JE, Terracciano A, et al. The trajectory of loneliness in response to COVID-19. Am Psychol. 2020:75(7):897-908. 
23 Rico-Uribe LA, Caballero FF, Martín-María N, Cabello M, Ayuso-Mateos JL, Miret M. Association of loneliness with all-cause mortality: A meta-analysis. Plos One. 2018;13:e0190033-e0190033.

24 Caspi A, Harrington H, Moffitt TE, Milne BJ, Poulton R. Socially isolated children 20 years later: risk of cardiovascular disease. Arch Pediatr Adolesc Med. 2006;160:805-811.

25 Seeman TE. Health promoting effects of friends and family on health outcomes in older adults. Am J Health Promot. 2000;14:362-370.

26 Holt-Lunstad J, Smith TB, Layton JB. Social Relationships and Mortality Risk: A Metaanalytic Review. PloS Med. 2010;7:e1000316.

27 Kiecolt-Glaser JK, Garner W, Speicher C, Penn GM, Holliday J, Glaser R. Psychosocial modifiers of immunocompetence in medical students. Psychosom Med. 1984;46(1):7-14.

28 Pressman SD, Cohen S, Miller GE, Barkin A, Rabin BS, Treanor JJ. Loneliness, social network size, and immune response to influenza vaccination in college freshmen. Health Psychol. 2005;24:297-306.

29 Steptoe A, Owen N, Kunz-Ebrecht SR, Brydon L. Loneliness and neuroendocrine, cardiovascular, and inflammatory stress responses in middle-aged men and women. Psychoneuroendocrinology. 2004;29:593-611.

30 Hawkley LC, Masi CM, Berry JD, Cacioppo JT. Loneliness is a unique predictor of agerelated differences in systolic blood pressure. Psychol Aging. 2006;21:152.

31 Hawkley LC, Thisted RA, Masi CM, Cacioppo JT. Loneliness predicts increased blood pressure: 5-year cross-lagged analyses in middle-aged and older adults. Psychol Aging. 2010;25:132.

32 Valtorta NK, Kanaan M, Gilbody S, Ronzi S, Hanratty B. Loneliness and social isolation as risk factors for coronary heart disease and stroke: systematic review and meta-analysis of longitudinal observational studies. Heart. 2016;102:1009-1016.

33 Hawkley LC, Thisted RA, Cacioppo JT. Loneliness predicts reduced physical activity: cross-sectional \& longitudinal analyses. Health Psychol. 2009;28:354.

34 Mason TB. Loneliness, eating, and body mass index in parent-adolescent dyads from the Family Life, Activity, Sun, Health, and Eating study. Pers Relatsh. 2020;27:420-432.

35 Lauder W, Mummery K, Jones M, Caperchione C. A comparison of health behaviours in lonely and non-lonely populations. Psychol Health Med. 2006;11:233-245.

36 Matthews T, Danese A, Gregory AM, Caspi A, Moffitt TE, Arseneault L. Sleeping with one eye open: loneliness and sleep quality in young adults. Psychol Med. 2017;47:2177-2186. 37 Segrin C, Burke TJ. Loneliness and Sleep Quality: Dyadic Effects and Stress Effects. Behav Sleep Med. 2015;13:241-254.

38 Ben Simon E, Walker MP. Sleep loss causes social withdrawal and loneliness. Nat Commun. 2018;9:3146.

39 Holwerda TJ, Deeg DJH, Beekman ATF, van Tilburg TG, Stek ML, Jonker C, et al. Feelings of loneliness, but not social isolation, predict dementia onset: results from the Amsterdam Study of the Elderly (AMSTEL). J Neurol Neurosurg Psychiatry. 2014;85(2):13542.

40 Wilson RS, Krueger KR, Arnold SE, Schneider JA, Kelly JF, Barnes LL, et al. Loneliness and risk of Alzheimer disease. Arch Gen Psychiatry. 2007;64(2):234-40.

41 Beutel ME, Klein EM, Brähler E, Reiner I, Jünger C, Michal M, et al. Loneliness in the general population: prevalence, determinants and relations to mental health. BMC Psychiatry. 2017;17:97.

42 Erzen E, Çikrikci Ö. The effect of loneliness on depression: A meta-analysis. Int J Soc Psychiatry. 2018;64:427-435.

43 Eres R, Lim MH, Lanham S, Jillard C, Bates G. Loneliness and emotion regulation: implications of having social anxiety disorder. Aust J Psychol. 2021;73:46-56.

44 Richardson T, Elliott P, Roberts R: Relationship between loneliness and mental health in students. J Public Ment Health. 2017;16(2):48-54.

45 Morr M, Lieberz J, Dobbelstein M, Philipsen A, Hurlemann R, Scheele D. Insula reactivity mediates subjective isolation stress in alexithymia. Sci Rep. 2021;11:1-9.

46 Åkerlind I, Hörnquist JO. Loneliness and alcohol abuse: A review of evidences of an interplay. Soc Sci Med. 1992;34:405-414. 
47 García-Montes JM, Zaldívar-Basurto F, López-Ríos F, Molina-Moreno A. The Role of Personality Variables in Drug Abuse in a Spanish University Population. Int J Ment Health Addict. 2009;7:475-487.

48 Bragard E, Giorgi S, Juneau P, Curtis BL. Loneliness and Daily Alcohol Consumption During the COVID-19 Pandemic. Alcohol Alcohol. 2021:agab056.

49 Liebke L, Bungert M, Thome J, Hauschild S, Gescher DM, Schmahl C, et al. Loneliness, social networks, and social functioning in borderline personality disorder. Personal Disord. 2017;8:349-356.

50 Hauschild S, Winter D, Thome J, Liebke L, Schmahl C, Bohus M, et al. Behavioural mimicry and loneliness in borderline personality disorder. Compr. Psychiatry. 2018;82:30-36.

51 Martens WH. Schizoid personality disorder linked to unbearable and inescapable loneliness. Eur J Psychiatry. 2010;24:38-45.

52 O'Connor M. A longitudinal study of PTSD in the elderly bereaved: Prevalence and predictors. Aging Ment Health. 2010;14:310-318.

53 Lee B, Youm Y. Social network effects on post-traumatic stress disorder (PTSD) in female North Korean immigrants. J Prev Med Public Health. 2011;44:191-200.

54 Scheele D, Lieberz J, Goertzen-Patin A, Engels C, Schneider L, Stoffel-Wagner B, Becker B. Trauma disclosure moderates the effects of oxytocin on intrusions and neural responses to fear. Psychother Psychosom. 2019;88:61-64.

55 Morr M, Noell J, Sassin D, Daniels J, Philipsen A, Becker B, et al. Lonely in the dark: trauma memory and sex-specific dysregulation of amygdala reactivity to fear signals. bioRxiv 2021. https://doi.org/10.1101/2021.11.16.468598 .

56 Stahn AC, Gunga H-C, Kohlberg E, Gallinat J, Dinges DF, Kühn S. Brain changes in response to long Antarctic expeditions. N Engl J Med. 2019;381:2273-2275.

57 Biggio F, Mostallino MC, Talani G, Locci V, Mostallino R, Calandra G, et al. Social enrichment reverses the isolation-induced deficits of neuronal plasticity in the hippocampus of male rats. Neuropharmacol. 2019;151:45-54.

58 Bickart KC, Wright Cl, Dautoff RJ, Dickerson BC, Barrett LF. Amygdala volume and social network size in humans. Nat Neurosci. 2011;14:163-164.

59 Lin C, Keles U, Tyszka JM, Gallo M, Paul L, Adolphs R. No strong evidence that social network index is associated with gray matter volume from a data-driven investigation. Cortex. 2020;125:307-317.

60 Becker B, Mihov Y, Scheele D, Kendrick KM, Feinstein JS, Matusch A et al. Fear processing and social networking in the absence of a functional amygdala. Biol Psychiatry. 2012;72:70-77.

61 Mihov Y, Kendrick KM, Becker B, Zschernack J, Reich H, Maier W, et al. Mirroring fear in the absence of a functional amygdala. Biol Psychiatry. 2013;73:e9-e11.

62 Scheele D, Zimbal S, Feinstein JS, Delis A, Neumann C, Mielacher C, et al. Treatmentresistant depression and ketamine response in a patient with bilateral amygdala damage. Am J Psychiatry. 2019;176:982-986.

63 Kiesow H, Dunbar RI, Kable JW, Kalenscher T, Vogeley K, Schilbach L, et al. 10,000 social brains: sex differentiation in human brain anatomy. Sci Adv. 2020;6:eaaz1170.

64 Spreng RN, Dimas E, Mwilambwe-Tshilobo L, Dagher A, Koellinger P, Nave G, et al. The default network of the human brain is associated with perceived social isolation. Nat Commun. 2020;11:1-11.

65 Kanai R, Bahrami B, Duchaine B, Janik A, Banissy MJ, Rees G. Brain structure links loneliness to social perception. Curr Biol. 2012;22:1975-1979.

66 Lieberz J, Shamay-Tsoory SG, Saporta N, Kanterman A, Gorni J, Esser T, et al. Behavioral and neural dissociation of social anxiety and loneliness. medRxiv 2021:2021.2008.2025.21262544.

67 Cacioppo JT, Hawkley LC. Perceived social isolation and cognition. Trends Cogn Sci. 2009;13:447-454.

68 Courtney AL, Meyer ML. Self-Other Representation in the Social Brain Reflects Social Connection. J Neurosci. 2020;40:5616-5627. 
69 Lieberz J, Shamay-Tsoory SG, Saporta N, Esser T, Kuskova E, Stoffel-Wagner B, et al. Loneliness and the social brain: how perceived social isolation impairs human interactions. bioRxiv 2021:2021.2003.2003.433569.

70 Eisenberger NI. The pain of social disconnection: examining the shared neural underpinnings of physical and social pain. Nat Rev Neurosci. 2012;13(6):421-34.

71 Arnold AJ, Winkielman P, Dobkins K: Interoception and Social Connection. Front Psychol. 2019;10:2589.

72 Mwilambwe-Tshilobo L, Ge T, Chong M, Ferguson MA, Misic B, Burrow AL, et al. Loneliness and meaning in life are reflected in the intrinsic network architecture of the brain. Soc Cogn Affect Neurosci. 2019;14:423-433.

73 Zhou H-X, Chen X, Shen Y-Q, Li L, Chen N-X, Zhu Z-C, et al. Rumination and the default mode network: Meta-analysis of brain imaging studies and implications for depression. Neuroimage 2020;206:116287.

74 Saporta N, Scheele D, Lieberz J, Nevat M, Kanterman A, Hurlemann R, et al. Alone Again: Altered Activation in the Observation Execution System during Synchronization in High Loneliness Individuals. bioRxiv 2021:2021.2010.2017.464634.

75 Tian Y, Yang L, Chen S, Guo D, Ding Z, Tam KY, et al. Causal interactions in restingstate networks predict perceived loneliness. PloS One. 2017;12:e0177443.

76 von Mohr M, Kirsch LP, Fotopoulou A. Social touch deprivation during COVID-19: effects on psychological wellbeing and craving interpersonal touch. R Soc Open Sci. 2021;8:210287-210287.

77 Saporta N, Peled-Avron L, Scheele D, Lieberz J, Hurlemann R, Shamay-Tsoory SG. Touched by Loneliness - How Loneliness Impacts the Response to Observed Human Touch: a tDCS Study. Soc Cogn Affect Neurosci. 2021:nsab122.

78 Blomkvist A, Hofer M. Olfactory Impairment and Close Social Relationships. A Narrative Review. Chem Senses. 2021;46:bjab037.

79 Maier A, Heinen-Ludwig L, Güntürkün O, Hurlemann R, Scheele D. Childhood Maltreatment Alters the Neural Processing of Chemosensory Stress Signals. Front Psychiatry. 2020;11

80 Cacioppo JT, Ernst JM, Burleson MH, McClintock MK, Malarkey WB, Hawkley LC, et al. Lonely traits and concomitant physiological processes: the MacArthur social neuroscience studies. Int J Psychophyiol. 2000;35:143-154.

81 Baumeister RF, DeWall CN, Ciarocco NJ, Twenge JM. Social exclusion impairs selfregulation. J Pers Soc Psychol. 2005;88:589.

82 Twenge JM, Baumeister RF, Tice DM, Stucke TS. If you can't join them, beat them: effects of social exclusion on aggressive behavior. J Pers Soc Psychol. 2001;81:1058.

83 Layden EA, Cacioppo JT, Cacioppo S, Cappa SF, Dodich A, Falini A, et al. Perceived social isolation is associated with altered functional connectivity in neural networks associated with tonic alertness and executive control. Neuroimage. 2017;145:58-73.

84 Cacioppo JT, Norris CJ, Decety J, Monteleone G, Nusbaum H. In the eye of the beholder: individual differences in perceived social isolation predict regional brain activation to social stimuli. J Cogn Neurosci. 2009;21:83-92.

85 D'Agostino AE, Kattan D, Canli T. An fMRI study of loneliness in younger and older adults. Soc Neurosci. 2019;14:136-148.

86 Schalbroeck R, van Velden FHP, de Geus-Oei L-F, Yaqub M, van Amelsvoort T, Booij $\mathrm{J}$, et al. Striatal dopamine synthesis capacity in autism spectrum disorder and its relation with social defeat: an [(18)F]-FDOPA PET/CT study. TransI Psychiatry. 2021;11:47-47.

87 Yin J, Lassale C, Steptoe A, Cadar D. Exploring the bidirectional associations between loneliness and cognitive functioning over 10 years: the English longitudinal study of ageing. Int J Epidemiol. 2019;48:1937-1948.

88 McHugh Power JE, Steptoe A, Kee F, Lawlor BA. Loneliness and social engagement in older adults: A bivariate dual change score analysis. Psychol Aging. 2019;34:152.

89 Rosenstreich E, Margalit M. Loneliness, mindfulness, and academic achievements: A moderation effect among first-year college students. Open Psychol J. 2015;8 
90 Gao M, Shao R, Huang C-M, Liu H-L, Chen Y-L, Lee S-H, et al. The relationship between loneliness and working-memory-related frontoparietal network connectivity in people with major depressive disorder. Behav Brain Res. 2020;393:112776.

91 Zagic D, Wuthrich VM, Rapee RM, Wolters N. Interventions to improve social connections: a systematic review and meta-analysis. Soc Psychiatry Psychiatr Epidemiol. 2021:1-22.

92 Jarvis MA, Padmanabhanunni A, Chipps J. An Evaluation of a Low-Intensity Cognitive Behavioral Therapy mHealth-Supported Intervention to Reduce Loneliness in Older People. Int J Environ Res Public Health. 2019;16:1305.

93 Theeke LA, Mallow JA, Moore J, McBurney A, Rellick S, VanGilder R. Effectiveness of LISTEN on loneliness, neuroimmunological stress response, psychosocial functioning, quality of life, and physical health measures of chronic illness. Int J Nurs Sci. 2016;3:242-251.

94 Lindsay EK, Young S, Brown KW, Smyth JM, Creswell JD. Mindfulness training reduces loneliness and increases social contact in a randomized controlled trial. Proc Natl Acad Sci USA. 2019;116:3488-3493.

95 Creswell JD, Irwin MR, Burklund LJ, Lieberman MD, Arevalo JMG, Ma J, et al. Mindfulness-Based Stress Reduction training reduces loneliness and pro-inflammatory gene expression in older adults: A small randomized controlled trial. Brain Behav Immun. 2012;26:1095-1101.

96 Zhang N, Fan F-M, Huang S-Y, Rodriguez MA. Mindfulness training for loneliness among Chinese college students: A pilot randomized controlled trial. Int $\mathrm{J}$ Psychol. 2018;53:373-378.

97 Choi NG, Pepin R, Marti CN, Stevens CJ, Bruce ML. Improving Social Connectedness for Homebound Older Adults: Randomized Controlled Trial of Tele-Delivered Behavioral Activation Versus Tele-Delivered Friendly Visits. Am J Geriatr Psychiatry. 2020;28(7):698-708. 98 Shao R, Liu H-L, Huang C-M, Chen Y-L, Gao M, Lee S-H, et al. Loneliness and depression dissociated on parietal-centered networks in cognitive and resting states. Psychol Med. 2020;50:2691-2701.

99 Quintana DS, Lischke A, Grace S, Scheele D, Ma Y, Becker B. Advances in the field of intranasal oxytocin research: lessons learned and future directions for clinical research. Mol Psychiatry. 2021;26:80-91.

100 Xin F, Zhou X, Dong D, Zhao Z, Yang X, Wang Q, et al. Oxytocin Differentially Modulates Amygdala Responses during Top-Down and Bottom-Up Aversive Anticipation. Adv Sci. 2020;7:2001077.

101 Liu C, Lan C, Li K, Zhou F, Yao S, Xu L, et al. Oxytocinergic modulation of threatspecific amygdala sensitization in humans is critically mediated by serotonergic mechanisms. Biol Psychiatry: Cogn Neurosci Neuroimaging 2021;6(11):1081-1089.

102 Yao S, Zhao W, Geng Y, Chen Y, Zhao Z, Ma X, et al. Oxytocin facilitates approach behavior to positive social stimuli via decreasing anterior insula activity. Int J Neuropsychopharmacol. 2018;21:918-925.

103 Scheele D, Kendrick KM, Khouri C, Kretzer E, Schläpfer TE, Stoffel-Wagner B, et al. An oxytocin-induced facilitation of neural and emotional responses to social touch correlates inversely with autism traits. Neuropsychopharmacol. 2014;39:2078-2085.

104 Gozzi M, Dashow EM, Thurm A, Swedo SE, Zink CF. Effects of oxytocin and vasopressin on preferential brain responses to negative social feedback. Neuropsychopharmacol. 2017;42:1409-1419.

105 Zhuang Q, Zheng X, Becker B, Lei W, Xu X, Kendrick KM. Intranasal vasopressin like oxytocin increases social attention by influencing top-down control, but additionally enhances bottom-up control. Psychoneuroendocrinology. 2021;133:105412.

106 Juan K, Zhang Y, Zhou F, Gao Z, Yao S, Zhao W, et al. Anxiolytic effects of chronic intranasal oxytocin on neural responses to threat are dose-frequency dependent. bioRxiv 2021 https://doi.org/10.1101/2021.04.20.440539 .

107 Spengler FB, Schultz J, Scheele D, Essel M, Maier W, Heinrichs M, et al. Kinetics and dose dependency of intranasal oxytocin effects on amygdala reactivity. Biol Psychiatry. 2017;82:885-894. 
108 Liu C, Huang Y, Chen L, Yu R. Lack of Evidence for the Effect of Oxytocin on Placebo Analgesia and Nocebo Hyperalgesia. Psychother Psychosom. 2020;89:185-188.

109 Zhao W, Becker B, Yao S, Ma X, Kou J, Kendrick KM. Oxytocin enhancement of the placebo effect may be a novel therapy for working memory impairments. Psychother Psychosom. 2019;88:125-127.

110 Colloca L, Pine DS, Ernst M, Miller FG, Grillon C. Vasopressin boosts placebo analgesic effects in women: a randomized trial. Biol Psychiatry. 2016;79:794-802.

111 Ma X, Zhao W, Luo R, Zhou F, Geng Y, Xu L, et al. Sex-and context-dependent effects of oxytocin on social sharing. Neuroimage. 2018;183:62-72.

112 Lieberz J, Scheele D, Spengler FB, Matheisen T, Schneider L, Stoffel-Wagner B, et al. Kinetics of oxytocin effects on amygdala and striatal reactivity vary between women and men. Neuropsychopharmacol. 2020;45:1134-1140.

113 Coenjaerts M, Trimborn I, Adrovic B, Stoffel-Wagner B, Cahill L, Philipsen A, et al. Estradiol and oxytocin modulate sex differences in hippocampal reactivity and episodic memory. bioRxiv $2021 \mathrm{https}: / /$ doi.org/10.1101/2021.11.22.469500 . 


\section{Figures}

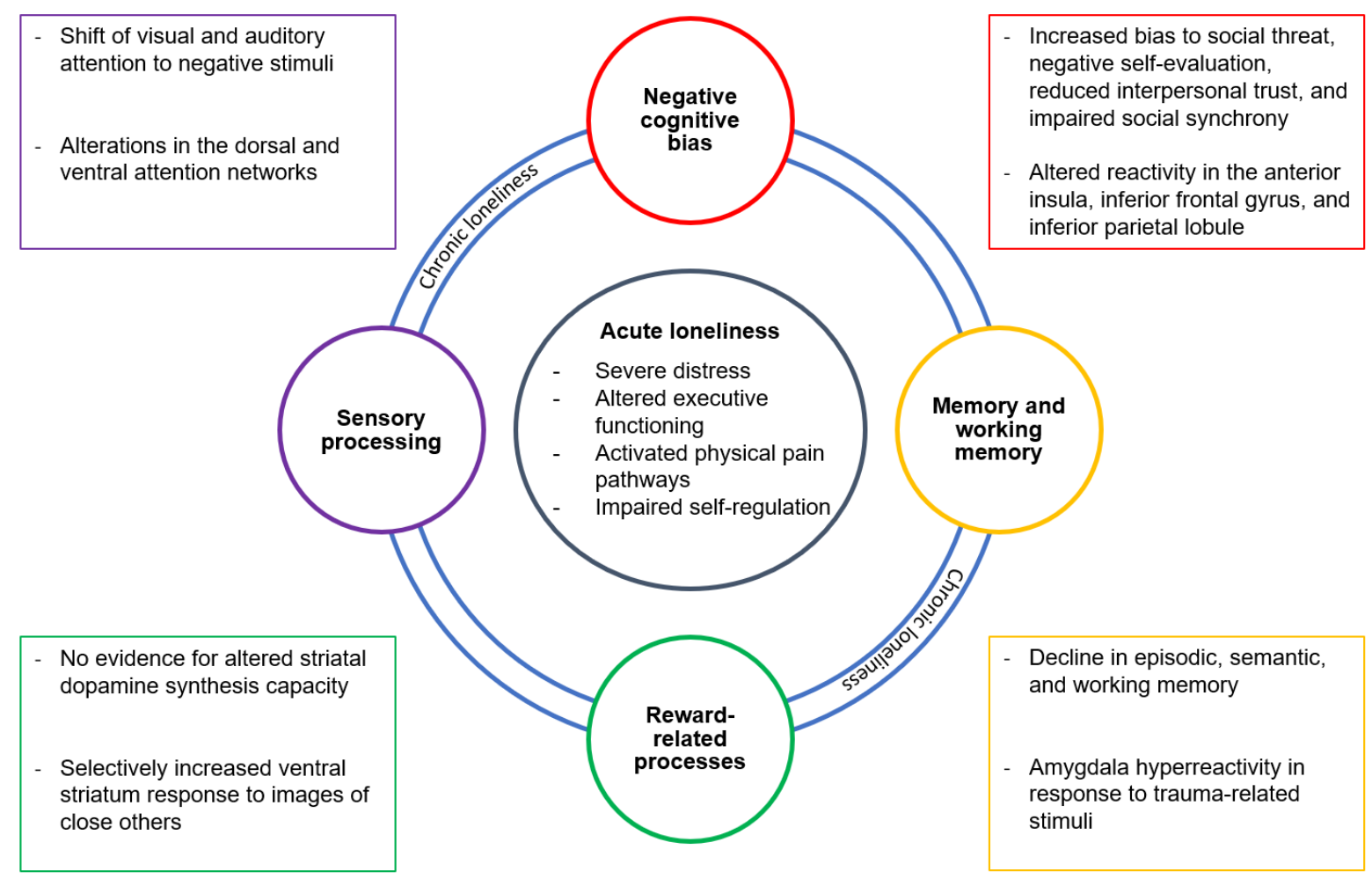

Fig. 1. Theoretical model illustrating the impact of acute and chronic loneliness. Acute effects of loneliness are shown in the inner circle. Chronic loneliness may affect functional domains which are illustrated in the outer circles. Exemplary findings for the domains are listed in the boxes: negative cognitive biases (red), memory and working memory (yellow), rewardrelated processes (green), and sensory processing (purple). 


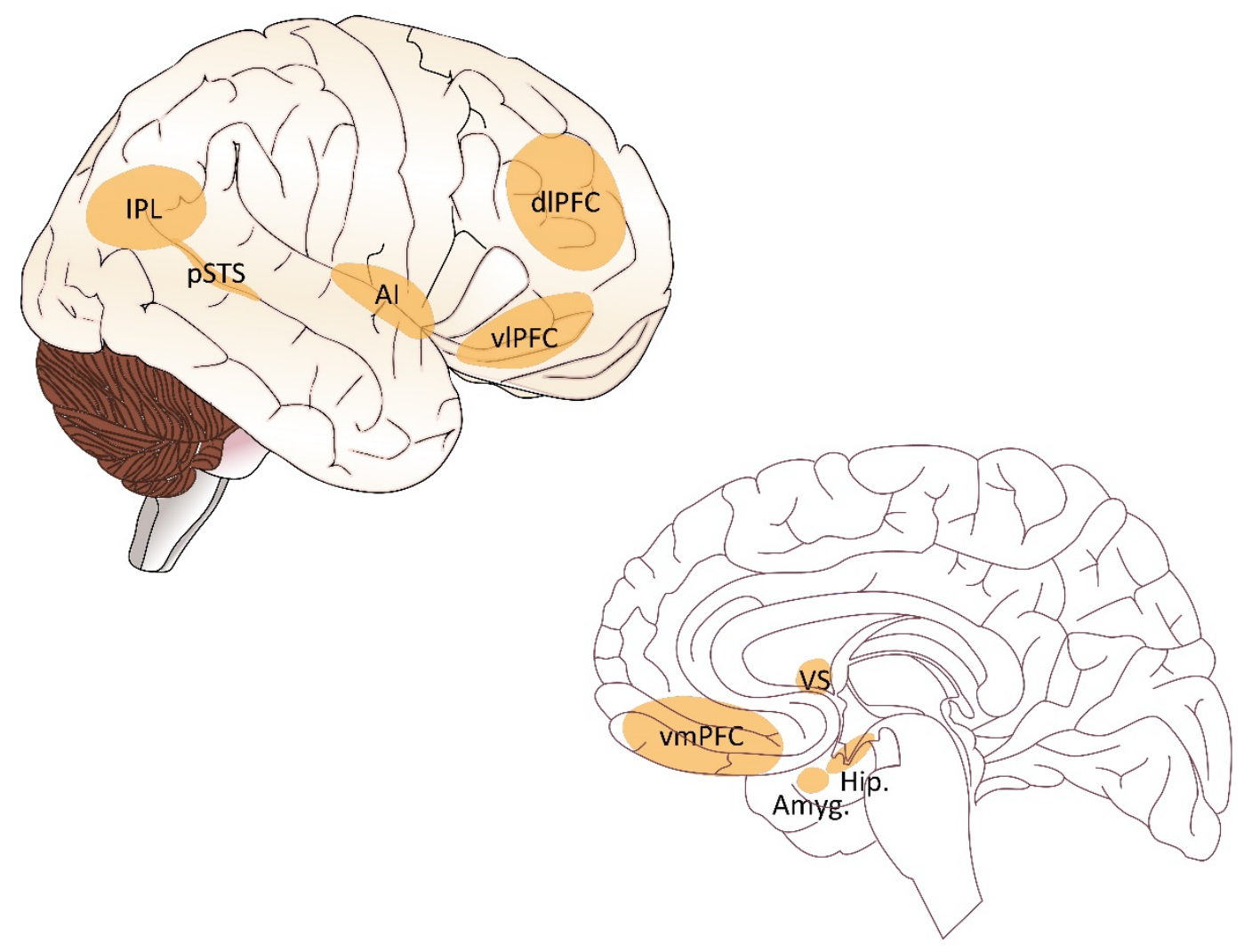

Fig. 2 Illustration of brain areas involved in loneliness. Chronic loneliness has been associated with functional and structural changes in various neural circuits of social and affective brain systems, including limbic regions such as the amygdala, hippocampus and the anterior insula, as well as striatal, prefrontal, and temporal regions Abbreviations: Amyg, amygdala; dIPFC, dorsolateral prefrontal cortex; Hip, hippocampus; IPL, inferior parietal lobule; AI, anterior insula, VS, ventral striatum; vIPFC, ventrolateral prefrontal cortex; vmPFC, ventromedial prefrontal cortex, pSTS, posterior superior temporal sulcus. Source of the brain template picture used to display the brain regions from https://scidraw.io/ (shared under the creative commons license CC-BY license). 